\title{
Computational modeling of brain tumors: discrete, continuum or hybrid?
}

\author{
Zhihui Wang · Thomas S. Deisboeck
}

Received: 21 March 2008 / Accepted: 25 March 2008 / Published online: 26 June 2008

(C) Springer Science+Business Media B.V. 2008

\begin{abstract}
In spite of all efforts, patients diagnosed with highly malignant brain tumors (gliomas), continue to face a grim prognosis. Achieving significant therapeutic advances will also require a more detailed quantitative understanding of the dynamic interactions among tumor cells, and between these cells and their biological microenvironment. Datadriven computational brain tumor models have the potential to provide experimental tumor biologists with such quantitative and cost-efficient tools to generate and test hypotheses on tumor progression, and to infer fundamental operating principles governing bidirectional signal propagation in multicellular cancer systems. This review highlights the modeling objectives of and challenges with developing such in silico brain tumor models by outlining two distinct computational approaches: discrete and continuum, each with representative examples. Future directions of this integrative computational neuro-oncology field, such as hybrid multiscale multiresolution modeling are discussed.
\end{abstract}

Keywords Brain tumor · Agent-based model $\cdot$ Cellular automata $\cdot$ Continuum · Multi-scale

$\begin{array}{ll}\text { Abbreviations } \\ \text { ABM } & \text { Agent-based model } \\ \text { CA } & \text { Cellular automata } \\ \text { EGFR } & \text { Epidermal growth factor receptor } \\ \text { ECM } & \text { Extracellular matrix } \\ \text { GBM } & \text { Glioblastoma } \\ \text { MRI } & \text { Magnetic resonance imaging } \\ \text { PLC } \gamma & \text { Phopholipase C } \gamma \\ \text { ROI } & \text { Region of interest }\end{array}$

Z. Wang · T. S. Deisboeck $(\varangle)$

Complex Biosystems Modeling Laboratory, Harvard-MIT (HST) Athinoula A. Martinos Center for Biomedical Imaging, Massachusetts General Hospital-East, 2301, Building 149, 13th Street, Charlestown, MA 02129, USA

e-mail: deisboec@helix.mgh.harvard.edu 
2D Two-dimensional

3D Three-dimensional

\section{Introduction}

There are two basic types of brain tumors, i.e. primary tumors and secondary or metastatic brain tumors. Primary brain tumors arise in the brain, and here most often from its supporting astrocytes or glia cells (hence the terminology 'astrocytoma' or 'glioma') and generally do not spread outside the brain tissue; on the contrary, metastatic brain tumors originate elsewhere in the body such as in the lung or skin before disseminated cancer satellite cells spread also to the brain. Carcinogenesis in the brain, much like elsewhere in the body, is a complex multistage process that originates from genetic changes, distortion of the cell cycle, and loss of apoptosis [1], and proceeds to angiogenesis, and extensive local infiltration and invasion [51]. In the United States, for the year 2007 alone, it was estimated that there were 20,500 new cases of (both primary and secondary) brain tumors, and 12,740 deaths related to this disease [37]. Brain tumors are still relatively insensitive to conventional cancer treatments, including radiation and chemotherapy [36]. Despite advances in recent targeted anticancer therapies, the clinical outcome in treating malignant brain tumors remains disappointing [60] with less than $30 \%$ of recurrent glioblastoma (GBM; the most aggressive form of gliomas) patients surviving without further progression six months after treatment [9]. This is mainly a result of the tumor's extensive infiltrative behavior, its rapid development of treatment resistance due to its inherent genetic and epigenetic heterogeneity, and the difficulties the so called blood-brain barrier poses for delivery of therapeutic compounds $[7,43]$.

Cellular and microenvironmental factors along with the underlying processes at the molecular level act as regulators of tumor growth and invasion [24,27]. Tumor cells bi-directionally communicate with their microenvironment: they not only respond to various external cues but also impact the environment by e.g. producing (auto- and paracrine) signals and degrading the neighboring tissue with proteases [34]. However, despite a vast amount of qualitative findings, conventional cancer research has made few gains in exploring the quantitative relationship between these very complicated intra- and intercellular signaling processes and the behavior they trigger on the microscopic and macroscopic scales [54]. It is here where we and others argue that systems biology [42] can provide useful insights, which may eventually promote the development of new cancer diagnostic and therapeutic techniques. While still in its beginning, systems biology has so far focused primarily on the single-cell level [2]. However, the usefulness of computational modeling and simulation, combined with experiment, is being increasingly recognized for exploring the dynamics at a multi-cell or tissue level of a variety of biological systems within a temporal, spatial and physiological context [8].

To date, computational modeling works have produced preliminary quantifications of the links between cell-cell and cell-extracellular matrix (ECM) interactions, cell motility, and local concentration of cell substrates. Already, this sprawling interdisciplinary field draws increasing attention from an experimental and clinical as well as pharmaceutical perspective $[14,21,31]$. A better understanding of the inherent complexity of these cancer systems requires intensified interdisciplinary research in which the next iteration of innovative computational models, informed by and continuously revised with experimental data, will play an important role of guiding experimental interpretation and design in going forward [29]. Here, we discuss first objectives of and challenges with modeling brain tumors mathematically and 
computationally and then briefly review some recent developments in using two distinct in silico $^{1}$ approaches.

\section{In silico brain tumor modeling: objectives \& challenges}

As for other in silico oncology efforts, the main objective of modeling brain tumors is to design and develop powerful simulation platforms capable of (1) providing a realistic mathematical representation and systematic treatment of the complexity of experimentally observed cancer phenomena, across the scales of interest and within its distinct biological context, (2) generating experimentally testable hypotheses to guide wet-lab research and help evaluate the algorithms, and finally (3) integrating any number of distinct data qualities (e.g., serum markers, genomics, phospho-proteomics and magnetic resonance images) into these modeling algorithms in an effort to predict tumor growth patterns eventually also in a patientspecific context. To achieve such ambitious goals, a computational brain tumor model should be able to (i) quantitatively clarify the characteristics of a set of various basic cancer phenotypes (e.g., proliferation, invasion, and angiogenesis) at different scales, and (ii) to assess the impact of the microenvironmental cues on these cell phenotypes. Finally, we argue that (iii) an advanced brain tumor model should eventually be extended to the molecular level in that it explicitly includes the combinational effects of oncogenes [15] and tumor suppressor genes [11] on the aforementioned microscopic phenotypes.

There are several key challenges confronting a computational tumor biologist in developing any such model. These include: 1) Selection of modeling scale(s). Choosing the appropriate scale is the first critical step, usually guided by both the data available and the area of expertise of the investigator. Also, if a model is designed to be composed of different scales, then how to link these scales in a way supported by data is another non-trivial step. For example, GBM cells exhibit a variety of point mutations (molecular level) [35] that can affect microvascular remodeling (microscopic level) which in turn impacts tumor size, shape, and composition (macroscopic level) [33]. To date, while some brain tumor modeling studies have dealt with the interaction of processes between cellular and macroscopic levels (for a recent review, see [54]), only very few works made an attempt to quantitatively establish the relationship between the molecular and cellular levels. 2) Level of complexity versus computational cost. Generally, it holds that the more detailed a model, the more parameters are involved and thus the higher the computational 'cost' of running the algorithm. As such, for the time being, it is a compromise between the biological complexity to be represented and the computational resources this would require. Given the ever increasing amount of data available, scalability becomes an issue of paramount interest when deciding on the applicability of any such in silico tool in a clinical setting. 3) Tumor boundary definition. Defining the degree of diffuse invasion of tumor cells into the surrounding brain tissue remains difficult regardless of advancements in conventional medical imaging [69]. While some algorithms have made progress on translating tumor and surrounding tissue information from patient imaging data to the coordinate system of the models with finite element methods [16,49], there is still a long way to go towards accurately predicting where and when what number of the currently still invisible but surely existent mobile tumor cells spread into the adjacent healthy brain tissue.

1 In silico refers to experiments carried out entirely using a computer as opposed to being conducted in a wet lab environment (see [47] for a brief review on differences between in silico and in vitro or in vivo studies). 
Available computational models have addressed these challenges in one form or another. The next section will detail current approaches with a focus on briefly reviewing some significant findings of representative models developed in the past few years, and highlight some research groups active at the forefront of this interdisciplinary field.

\section{Computational modeling approaches}

Two major types of modeling strategies currently exist in the computational tumor modeling community: discrete and continuum approaches. Discrete models can explicitly represent individual cells in space and time and easily incorporate biological rules (based on data or assumptions), such as defining cell-cell and cell-matrix interactions involved in both chemotaxis and haptotaxis for instance. However, these models are limited to relatively small numbers of cells due to the compute intense nature of the method, and as a result a typical discrete model is usually designed with a sub-millimeter or even lower domain size [70]. In contrast, continuum models, by describing e.g. extracellular matrix or the entire tumor tissue as continuum medium rather than at the resolution of individual cells, are able to capture larger-scale volumetric tumor growth dynamics at comparatively lesser computational cost. As a trade-off, continuum models lack sensitivity to small fluctuations or oscillatory behaviors of a tumor system at a smaller segment, such as tumor angiogenetic sprout branching [3]. That is a significant shortcoming as in some cases such small changes can be the leading cause in driving a nonlinear complex biosystem to a different state [10]. In the following, we will introduce the two approaches in more detail.

\subsection{Discrete modeling}

The two main, related discrete modeling methods extensively used in this context are cellular automata (CA) and agent-based model (ABM). A generic CA is a collection of cells on a grid of specified shape that synchronously evolves through a number of discrete time steps, according to an identical set of rules (applied to each single cell) based on the states of neighboring cells [71]. The grid can be implemented in any finite number of dimensions, and neighbors are a selection of cells relative to a given cell. In contrast, ABM asynchronously models phenomena as dynamical systems of interactions among and between agents and their environments $[12,32]$. An agent is any autonomous component that can interact or communicate with other components. Each biological cell is often represented as an agent in an $\mathrm{ABM}$, and indeed $\mathrm{ABM}$ is the natural extension of $\mathrm{CA}$. Because of the asynchronous characteristic, the ease of implementation and the richness of detail one can expect in exploring biosystem dynamics, ABM is an appealing choice for the simulation of tumors like glioma where the behavior and heterogeneity of the interacting cells cannot be safely reduced to some averaged, stylized or simple mechanism [66].

For instance, GBM growth dynamics in a three-dimensional (3D) environment have been successfully predicted using a CA model driven by four microscopic parameters (referring to cell-doubling time, nutritional needs of growth-arrested cells, nutritional needs of dividing cells, and effects of mechanical confinement pressure) $[39,40]$. This model was then used as the basis for a follow-up study to analyze a heterogeneous tumor by introducing a distinct subpopulation of tumor cells that exhibit a growth advantage [38]. The results showed that changes even in a small subpopulation may lead to a drastically altered tumor growth behavior, suggesting that prognosis based on the assumption of a homogeneous tumor cell population can be markedly inaccurate. With a CA approach to study the effects of surgery 
plus chemotherapy on the evolution of a homogeneous and more realistic heterogeneous GBM mass, it was found that the spatial distribution of chemotherapeutic resistant cells is an important indicator of persistence and continued tumor growth [55]. One clinical implication gained from this study is that the shape of the reoccurring tumor may depend on the rate at which chemotherapy induces mutations. Since these previous iterations made oversimplifying assumptions on tumor vascular and angiogenesis, a recent two-dimensional (2D) CA simulation tool [30] considered the processes of vessel co-option, regression and angiogenesis in tumor growth; it enabled the researchers to study the growth of a primary neoplasm from a small mass of cells to a macroscopic tumor mass, and to simulate how mutations affecting the angiogeneic response subsequently impact tumor development.

To investigate highly malignant brain tumors as complex, dynamic, and self-organizing biosystems [20], Deisboeck and co-workers have been focusing on the development of ABMs simulating tumor properties across multiple scales in time and space. First, the spatio-temporal expansion of virtual glioma cells in a 2D microscopic setup and the relationship between rapid growth and extensive tissue infiltration were investigated $[44,45]$. These earlier works reported a phase transition leading to the emergence of two distinct spatio-temporal patterns: a) a small number of larger tumor cell clusters exhibiting rapid spatial expansion but shorter overall lifetime of the tumor system, and b) many small clusters with longer lifetime but the tradeoff of a slower velocity of expansion, depending on different implicit chemotactic search strategies. Subsequently, by incorporating a molecular scale in the form of a simplified representation of the epidermal growth factor receptor (EGFR) signaling pathway (important for epithelial cancers in general, and for highly malignant brain tumors in particular [46]), the model was extended to capture tumor growth dynamics to a degree of any specific pathway component [5,6]. Some intriguing, testable hypotheses have been generated in terms of how molecular profiles of individual glioma cells impact the cellular phenotype and how such single-cell decisions can potentially affect the dynamics of the entire tumor system. Most recently, an explicit cell cycle description was introduced to the model and brain tumor growth dynamics were examined in a 3D context with a more complicated ECM representation at the microscopic scale [72]. Together, these works have provided a computational paradigm for simulating brain tumors from the molecular scale up to the cellular level and beyond. It should be noted that in these works some environmental parameters, such as growth factors, nutrient, and oxygen tension, were expressed with a continuum term. Another contribution of the works by Deisboeck and co-workers is that, based on available data $[23,48]$, they propose employing, as an example, an EGFR-downstream protein, phospholipase $\mathrm{C} \gamma$ (PLC $\gamma$ ), to determine two phenotypic traits, i.e. cell proliferation and migration, by comparing the rate of change of its molecular-level concentration with a predefined threshold. That is, a glioma cell becomes eligible to 1) migrate if the range of change of PLC $\gamma$ exceeds the threshold, and 2) proliferate if the range of change of PLC $\gamma$ is below that set threshold, yet above a noise threshold. More generic, the change in the concentration of a pathway component over time is calculated with a continuum element, i.e., according to the following differential equation:

$$
\frac{\mathrm{d} X i}{\mathrm{~d} t}=\alpha X i-\beta X i
$$

where $X i$ represents the concentration level of the $i$ th pathway component, and $\alpha$ and $\beta$ are the reaction rates of producing and consuming $X i$, respectively. Figure 1 shows a series of simulation results produced by the model [6], explaining how tumor growth dynamics at the cellular level can be related to alterations at the molecular level. This algorithm is flexible so that it can accommodate the governing, physical requirements of other cancer types, such as non-small cell lung cancer [67], which demonstrates the versatility of this design concept. 


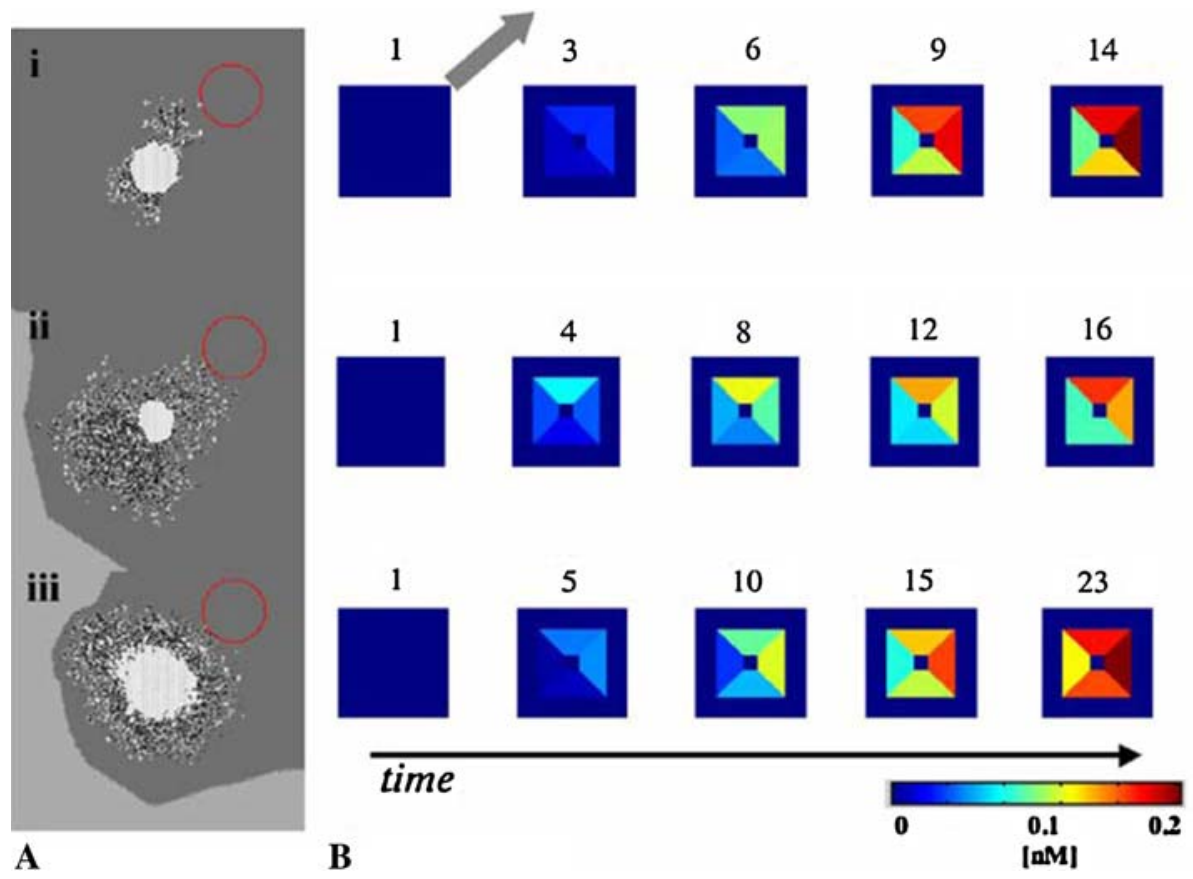

Fig. 1 (a) 2D cross-section of a tumor spheroid, for three different human glioma cell lines, from top to bottom: D-263 MG, D-247 MG, and D-37 MG. Each simulation was terminated when the first tumor cell reached the edge of a (red) nutrient source (representing an orthograde cut cerebral blood vessel) located in the north-east quadrant of the lattice. (b) Polarization of the molecular concentration profiles of the EGFR-pathway downstream component PLC $\gamma$ in the first cell, at five consecutive time points. A qualitatively similar PLC $\gamma$ polarization pattern emerges in the three cell lines as higher concentrations of PLC $\gamma$ eventually accumulate in the apical part of the cell that faces nutrient abundance. Adapted from [6]

It is noteworthy that some efforts employ techniques analogous to ABM to study the clinical level of brain tumor behavior. A series of in silico studies on simulating a GBM response to radiotherapy, considering vasculature and oxygen supply, has been conducted $[22,58,59]$. While in [59] tumor cells were considered individually, in the follow-up studies $[22,58]$, in an effort to overcome the extensive computational demand, cells were clustered into dynamic equivalence classes based on the mean cell cycle phase durations (G1, S, G2, and $\mathrm{M}$, see [41] for a review); that is, tumor response to radiotherapy was investigated on each cluster instead of on each individual cell. Moreover, for performing patient-specific in silico experiments as a means of chemotherapeutic treatment optimization, the same authors recently developed a four-dimensional simulation platform based on magnetic resonance imaging (MRI), histopathologic, and pharmacogenetic data, noting that the model's predictions were in good agreements with clinical practice [57]. Taken together, models from both Deisboeck's and Stamatakos' groups pioneered the integration of continuum elements into a discrete framework. To put this in perspective, we will detail a strict continuum approach in the following section.

\subsection{Continuum modeling}

Using a continuum approach, Cristini and co-workers have established a series of exploratory investigations on mathematical analysis of morphologic stability in growth and invasion of 
highly malignant gliomas $[17,18,25,26,53,56,73]$. They propose that tumor tissue dynamics can be simply regulated by two dimensionless parameters: one quantifies the competition between local cell proliferation (contributing to tumor mass growth) and cell adhesion (which tends to minimize the tumor surface area), while the other one represents tumor mass reduction related to cell death. The authors then tested the conditions for morphological stability for an independent set of experiments where the levels of growth factors and glucose were changed over a wide range in order to manipulate GBM cell proliferation and adhesion [25]. Most recently, they further confirmed that morphologic patterns of tumor boundary and infiltrative shapes of invasive tumors predicted by their models were in agreement with clinical histopathology samples of GBM from multiple patients [26]. Figure 2 shows a time-series result of the evolving tumor shape over a course of three months using this model. The authors claimed that their algorithm enabled the prediction of tumor morphology by quantifying the spatial diffusion gradients of cell substrates maintained by heterogeneous cell proliferation and an abnormal, constantly evolving vasculature. These models are based on reaction-diffusion equations (that govern variables such as tumor cell density, neovasculature, nutrient concentration, ECM, and matrix degrading enzymes) of the following generic form:

$$
v_{t}=-\nabla \cdot J+\Gamma_{+}-\Gamma_{-}
$$

where $v$ represents one of the evolving variables, $J$ is the flux, $\Gamma_{+}$and $\Gamma_{-}$are the sources and sinks with respect to variable $v$ (expansion formulas differ according to the variable investigated; see [26] for detail). This group's work showed that a continuum approach is capable of 1) accounting for a variety of invasive morphologies observed in tumors in vitro, in vivo, and in patients, 2) predicting different growth and invasion behaviors of tumors by calibrating model parameters, and 3) testing the hypothesized phenomenological relationships of tumor adhesion and proliferation that affect tissue-scale growth and morphology.

Several other groups have also been working on applying a continuum approach to the investigation of brain tumor behaviors. For instance, [61] developed a continuum model that incorporated the effects of heterogeneous brain tissue on diffusion and growth rates of glioma cells in an effort to represent asymmetries of the tumor boundaries. This basic work was then extended to examine the growth and invasion of gliomas in a 3D virtual brain refined from anatomical distributions of grey and white matter [62]. By allowing a motility coefficient to differ depending on the local tissue composition (so that glioma cells migrate more rapidly along white matter than in grey matter), the algorithm predicted sites of potential tumor recurrence to a degree beyond the limits of current medical imaging techniques. Interestingly, as supported by the results of this model, two independent factors, velocity of diametric expansion and initial tumor size at diagnosis, were indeed found to be statistically significant in a recent clinical survey on the prognostic evaluation of patients who harbor a grade II glioma [52]. Based on their previous studies [61,62,65], [63] also investigated the effects of chemotherapy on the spatio-temporal response of gliomas. By comparing the simulation results with MRI data of a glioma patient, it was suggested that differential delivery of the chemotherapeutic agent to the grey and white matter could successfully describe the clinical problems of shrinkage of the lesion in certain areas of the brain with continued growth in others. Another recent continuum model confirmed the effects of repeated immuno-suppression treatment (using different protocols) on the progression of glioma, and mathematically revealed the necessity of repeating such treatment in reducing the risk of recurrence [25]. Furthermore, by combining essential methods of two previous approaches [13,50], [68] were able to capture the spatio-temporal dynamics of drug transport and cell-death in a heterogeneous collection of glioma cells and normal brain cells. 

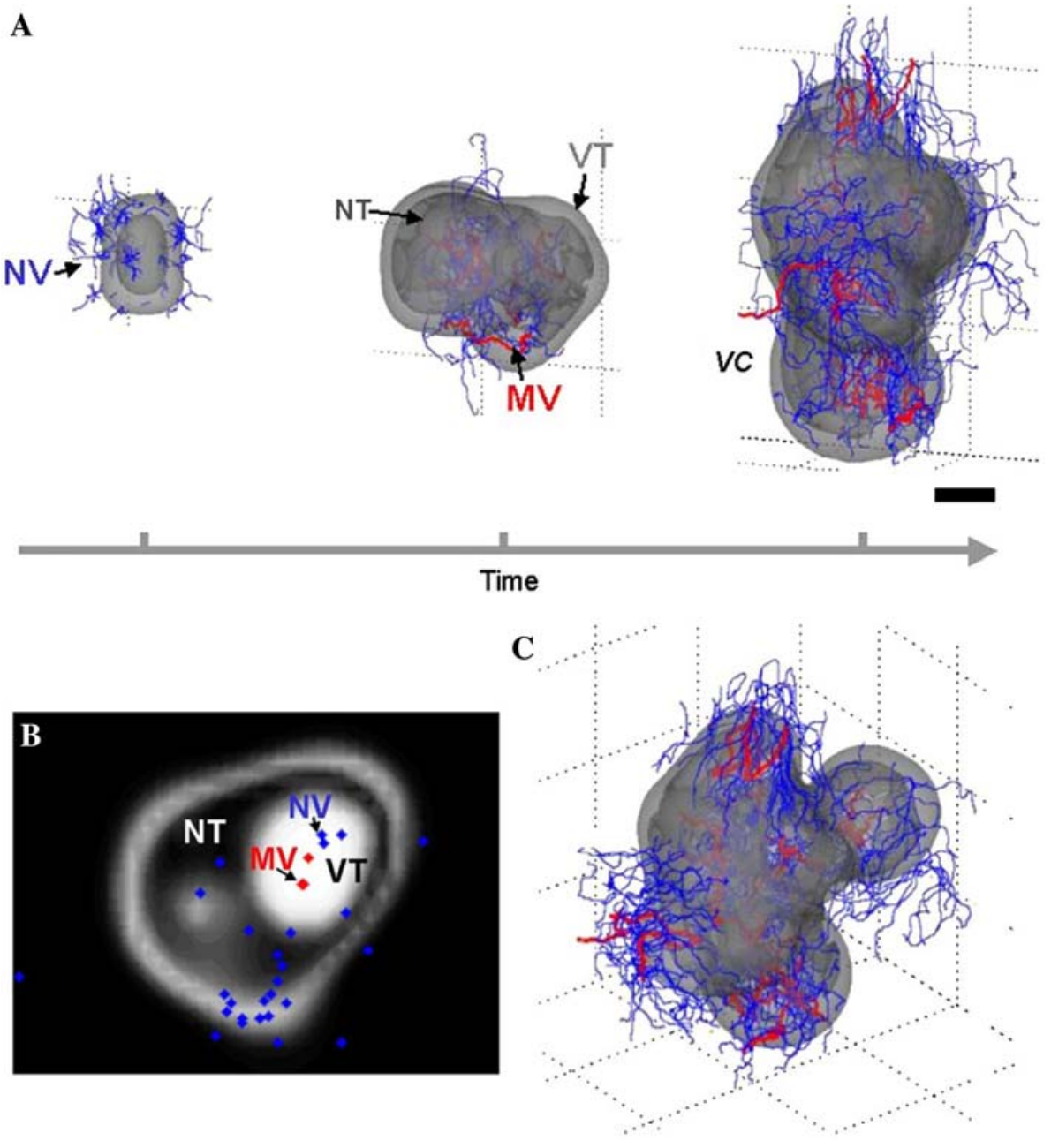

Fig. 2 (a) Time-series of the morphologic features of a growing GBM generated with a 3D model. The morphology is directly influenced by angiogenesis, vasculature maturation, and vessel co-option. The vessels labeled in red are capable of releasing nutrients, e.g., oxygen. (b) Histology-like section of the last frame of the simulation in (a) reveals viable tumor regions (white) surrounding necrotic tissue (dark). The viable region's thickness and extent of necrosis are strongly dependent on the diffusion gradients of oxygen/nutrient in the microenvironment. (c) Another view from the simulation shown in part A. Adapted from [26] with permission

\section{Conclusions and perspectives}

In recent years, computational cancer research has become a sprawling interdisciplinary field. This is a result of a number of contributing factors. Firstly, in contrast to conventional wet-lab experimental methods, such in silico models offer a powerful platform to reproducibly alter parameters and thus investigate their impact on the cancer system studied, at a rapid pace and in a cost-efficient way [42]. Secondly, computational models have demonstrated the ability of providing a useful hypothesis generating tool for refocusing experimental in vitro and in vivo works [54]. Thirdly, from a practical clinical perspective, computational modeling has already been applied, with some promise, to simulating the impact of chemotherapy, 
radiotherapy, and drug delivery on brain tumors [19]. Within this in silico oncology area, modeling and simulating malignant brain tumors is starting to emerge as a paramount driver for advancing technical developments en route to help addressing important scientific questions.

Demonstrated with examples from the literature, we have reviewed the two major mathematical modeling approaches, discussed their distinct merits and limitations in quantitatively studying brain tumor growth dynamics. In summary: While discrete models perform at the resolution of individual cells, which function independently through a set of behavioral rules that are inspired by biological facts if not fueled with real data, they are limited to a rather small number of cells or constituents. Conversely, continuum models can capture tumor growth at a collective scale that allows monitoring the expansion of a larger cluster of homogeneously behaving cells yet fail to register single cells, genes or proteins. Since both discrete and continuum modeling approaches have their own advantages and shortcomings (Table 1), and because quantifying the relationships between complex cancer phenomena at different scales is highly desirable, we and others have begun to move into the direction of hybrid modeling e.g., [4,58,67,72], or more appropriately, towards hybrid, multi-scale and multi-resolution algorithms as the next stage of cancer modeling in general, and brain tumor modeling in particular. While 'hybrid' refers to the integration of both discrete and continuum techniques, 'multi-resolution' means that cells at distinct topographic regions are treated differently in terms of the modeling approach applied. The overall strategy is clear: achieving discretely high resolution wherever and whenever necessary to maintain (or ideally, improve) the model's overall predictive power while at the same time reducing compute intensity as much as possible to allow for inclusion of sufficiently large datasets, and thus support scalability of the approach to clinically relevant levels. Figure 3 schematically describes the development of a 2D model using this novel strategy. Here, the MRI-demarked hypointense region within the tumor core, often comprised of a large fraction of apoptotic cells if not necrotic tissue, can arguably be described sufficiently as a rather homogenous population, thus at a lower resolution which allows employing a continuum module. Conversely, the highly active, gadolinium enhanced tumor surface supposedly thrives with a genetically and epigenetically heterogeneous population of cells that must at least in part be described discretely, and at the resolution of interconnected signaling pathways, to capture topographic areas that (e.g., with some probability, harbor an aggressive clone that) may impact overall growth patterns in the future. As the tumor grows, these high-resolution regions of interest (ROIs) and, thus the in silico modules representing them, will likely have to change dynamically (i.e., in size, number and location) to maintain or, better, improve predictive power

Table 1 Characteristics of discrete, continuum and hybrid brain tumor modeling approaches

\begin{tabular}{lll}
\hline Category & Characteristics & References \\
\hline Discrete & - Autonomous cells, with a set of rules governing their behavior & {$[39,40,45]$} \\
& - Capable to investigate tumor dynamics at a single cell level and below & \\
& - Limited to a comparably smaller scale due to prohibitive computational & \\
& costs & {$[18,26,64]$} \\
Continuum & - Describing tumor tissue as a continuum medium & \\
& - Capable to capture larger-scale volumetric tumor dynamics & \\
& - Computational cost efficiency & \\
& - Difficult to implement heterogeneous cell-cell and cell-environmental & \\
Hybrid & interaction, or molecular level dynamics & {$[5,6,57,58,72]$} \\
& - Applicable to both small- and large-scale models & \\
\hline
\end{tabular}




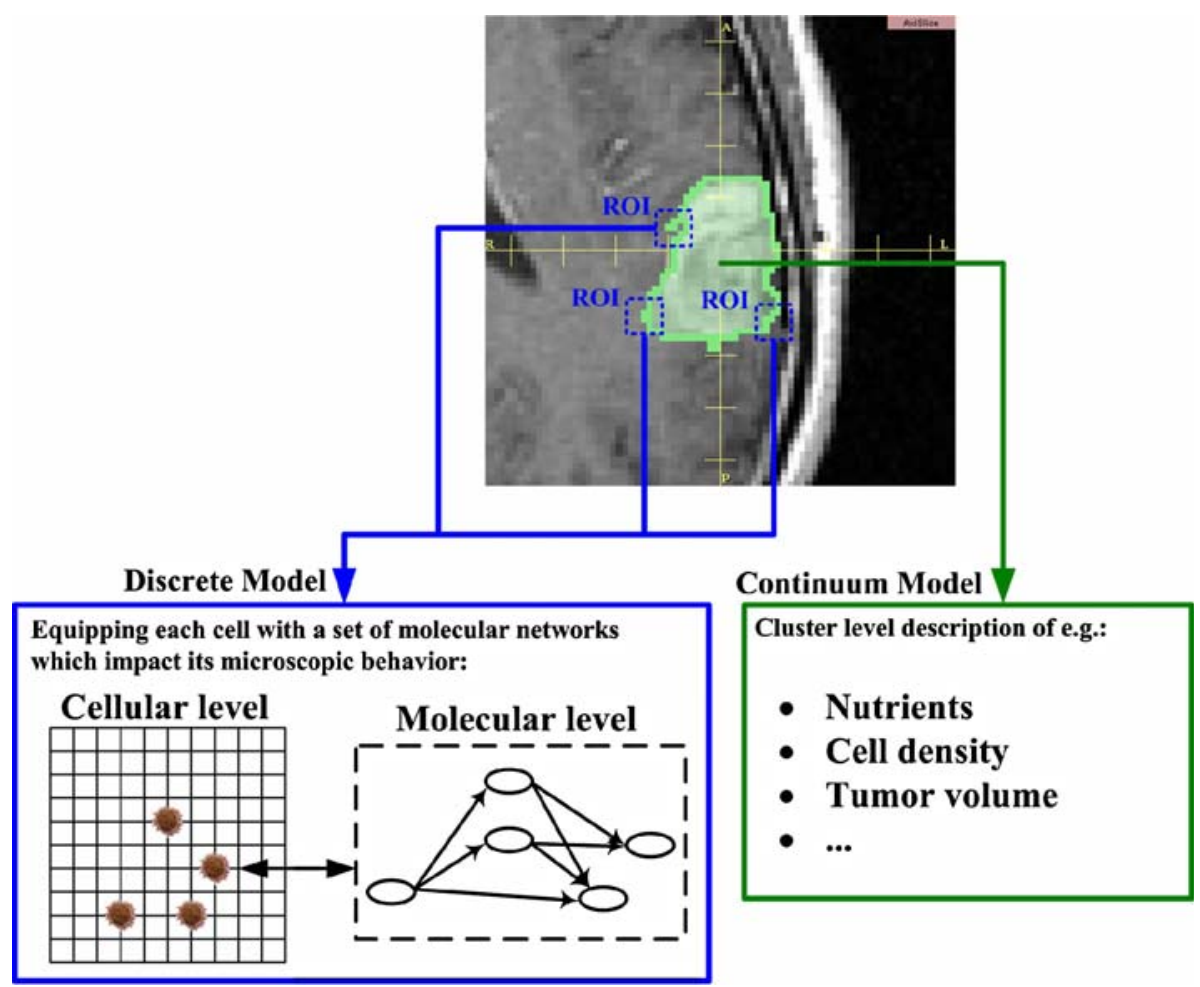

Fig. 3 Schematic illustration of a 2D brain tumor model using a hybrid, multi-scale and multi-resolution strategy. "ROI" represents a region of interest which refers to a higher modeling resolution desired and thus discrete-based technique used, versus the larger remaining volume (green) of the tumor tissue that is being modeled with a continuum-based approach. ROIs can be obtained e.g. by using finite elements and other numerical techniques $[3,17,73]$

while training on the patient-specific data set. Admittedly, much work needs to be done in this area to tackle the considerable challenges involved that range from data processing in 3D over time (where most computational savings would occur) to automated ROI placement and result driven, dynamic readjustment. However, eventually, such advanced in silico oncology approaches should be able to provide, on a clinical level, much needed quantitative insights into the dynamic cross-scale relationships that characterize these and other highly malignant tumors, and thus prove to become an effective and indispensable tool for personalized systems medicine in the near future.

Acknowledgements This work has been supported in part by NIH grant CA 113004 and by the HarvardMIT (HST) Athinoula A. Martinos Center for Biomedical Imaging and the Department of Radiology at Massachusetts General Hospital. We apologize to all colleagues whose works we could not cite due to space limitations.

\section{References}

1. Al-Hajj, M., Clarke, M.F.: Self-renewal and solid tumor stem cells. Oncogene 23, 7274-7282 (2004)

2. Albeck, J.G., MacBeath, G., White, F.M., Sorger, P.K., Lauffenburger, D.A., Gaudet, S.: Collecting and organizing systematic sets of protein data. Nat. Rev. Mol. Cell. Biol. 7, 803-812 (2006) 
3. Anderson, A.R., Chaplain, M.A.: Continuous and discrete mathematical models of tumor-induced angiogenesis. Bull. Math. Biol. 60, 857-899 (1998)

4. Anderson, A.R., Weaver, A.M., Cummings, P.T., Quaranta, V.: Tumor morphology and phenotypic evolution driven by selective pressure from the microenvironment. Cell 127, 905-915 (2006)

5. Athale, C., Mansury, Y., Deisboeck, T.S.: Simulating the impact of a molecular 'decision-process' on cellular phenotype and multicellular patterns in brain tumors. J. Theor. Biol. 233, 469-481 (2005)

6. Athale, C.A., Deisboeck, T.S.: The effects of EGF-receptor density on multiscale tumor growth patterns. J. Theor. Biol. 238, 771-779 (2006)

7. Badruddoja, M.A., Black, K.L.: Improving the delivery of therapeutic agents to CNS neoplasms: a clinical review. Front. Biosci. 11, 1466-1478 (2006)

8. Bailey, A.M., Thorne, B.C., Peirce, S.M.: Multi-cell agent-based simulation of the microvasculature to study the dynamics of circulating inflammatory cell trafficking. Ann. Biomed. Eng. 35, 916-936 (2007)

9. Ballman, K.V., Buckner, J.C., Brown, P.D., Giannini, C., Flynn, P.J., LaPlant, B.R., Jaeckle, K.A.: The relationship between six-month progression-free survival and 12-month overall survival end points for phase II trials in patients with glioblastoma multiforme. Neuro. Oncol. 9, 29-38 (2007)

10. Berg, O.G., Paulsson, J., Ehrenberg, M.: Fluctuations and quality of control in biological cells: zero-order ultrasensitivity reinvestigated. Biophys. J. 79, 1228-1236 (2000)

11. Blume-Jensen, P., Hunter, T.: Oncogenic kinase signalling. Nature 411, 355-365 (2001)

12. Bonabeau, E.: Agent-based modeling: methods and techniques for simulating human systems. Proc. Natl. Acad. Sci. USA 99(Suppl 3), 7280-7287 (2002)

13. Burgess, P.K., Kulesa, P.M., Murray, J.D., Alvord, E.C. Jr.: The interaction of growth rates and diffusion coefficients in a three-dimensional mathematical model of gliomas. J. Neuropathol. Exp. Neurol. 56, 704713 (1997)

14. Chaplain, M.A., McDougall, S.R., Anderson, A.R.: Mathematical modeling of tumor-induced angiogenesis. Annu. Rev. Biomed. Eng. 8, 233-257 (2006)

15. Cheng, J.Q., Lindsley, C.W., Cheng, G.Z., Yang, H., Nicosia, S.V.: The Akt/PKB pathway: molecular target for cancer drug discovery. Oncogene 24, 7482-7492 (2005)

16. Clatz, O., Sermesant, M., Bondiau, P.Y., Delingette, H., Warfield, S.K., Malandain, G., Ayache, N.: Realistic simulation of the 3-D growth of brain tumors in MR images coupling diffusion with biomechanical deformation. IEEE Trans. Med. Imaging 24, 1334-1346 (2005)

17. Cristini, V., Lowengrub, J., Nie, Q.: Nonlinear simulation of tumor growth. J. Math. Biol. 46, 191224 (2003)

18. Cristini, V., Frieboes, H.B., Gatenby, R., Caserta, S., Ferrari, M., Sinek, J.: Morphologic instability and cancer invasion. Clin. Cancer Res. 11, 6772-6779 (2005)

19. Deisboeck, T.S., Zhang, 1., Yoon, J., Costa, J.: In silico cancer modeling: Is ready for primetime? Nat. Clin. Pract. Oncol. (in press)

20. Deisboeck, T.S., Berens, M.E., Kansal, A.R., Torquato, S., Stemmer-Rachamimov, A.O., Chiocca, E.A.: Pattern of self-organization in tumour systems: complex growth dynamics in a novel brain tumour spheroid model. Cell Prolif. 34, 115-134 (2001)

21. Di Ventura, B., Lemerle, C., Michalodimitrakis, K., Serrano, L.: From in vivo to in silico biology and back. Nature 443, 527-533 (2006)

22. Dionysiou, D.D., Stamatakos, G.S., Uzunoglu, N.K., Nikita, K.S., Marioli, A.: A four-dimensional simulation model of tumour response to radiotherapy in vivo: parametric validation considering radiosensitivity, genetic profile and fractionation. J. Theor. Biol. 230, 1-20 (2004)

23. Dittmar, T., Husemann, A., Schewe, Y., Nofer, J.R., Niggemann, B., Zanker, K.S., Brandt, B.H.: Induction of cancer cell migration by epidermal growth factor is initiated by specific phosphorylation of tyrosine 1248 of c-erbB-2 receptor via EGFR. FASEB J. 16, 1823-1825 (2002)

24. Entschladen, F., Drell, T.L.t., Lang, K., Joseph, J., Zaenker, K.S.: Tumour-cell migration, invasion, and metastasis: navigation by neurotransmitters. Lancet Oncol. 5, 254-258 (2004)

25. Frieboes, H.B., Zheng, X., Sun, C.H., Tromberg, B., Gatenby, R., Cristini, V.: An integrated computational/experimental model of tumor invasion. Cancer Res. 66, 1597-1604 (2006)

26. Frieboes, H.B., Lowengrub, J.S., Wise, S., Zheng, X., Macklin, P., Bearer, E.L., Cristini, V.: Computer simulation of glioma growth and morphology. Neuroimage 37(Suppl 1), S59-S70 (2007)

27. Friedl, P., Wolf, K.: Tumour-cell invasion and migration: diversity and escape mechanisms. Nat. Rev. Cancer 3, 362-374 (2003)

28. Friedman, A., Tian, J.P., Fulci, G., Chiocca, E.A., Wang, J.: Glioma virotherapy: effects of innate immune suppression and increased viral replication capacity. Cancer Res. 66, 2314-2319 (2006)

29. Gatenby, R.A., Maini, P.K.: Mathematical oncology: cancer summed up. Nature 421, 321 (2003)

30. Gevertz, J.L., Torquato, S.: Modeling the effects of vasculature evolution on early brain tumor growth. J. Theor. Biol. 243, 517-531 (2006) 
31. Gilbert, D., Fuss, H., Gu, X., Orton, R., Robinson, S., Vyshemirsky, V., Kurth, M.J., Downes, C.S., Dubitzky, W.: Computational methodologies for modelling, analysis and simulation of signalling networks. Brief Bioinform. 7, 339-353 (2006)

32. Gilbert, N., Bankes, S.: Platforms and methods for agent-based modeling. Proc. Natl. Acad. Sci. USA 99(Suppl 3), 7197-7198 (2002)

33. Gilhuis, H.J., Bernse, H.J., Jeuken, J.W., Wesselin, P., Sprenger, S.H., Kerstens, H.M., Wiegant, J., Boerman, R.H.: The relationship between genetic aberrations as detected by comparative genomic hybridization and vascularization in glioblastoma xenografts. J. Neurooncol. 51, 121-127 (2001)

34. Hendrix, M.J., Seftor, E.A., Seftor, R.E., Kasemeier-Kulesa, J., Kulesa, P.M., Postovit, L.M.: Reprogramming metastatic tumour cells with embryonic microenvironments. Nat. Rev. Cancer 7, 246-255 (2007)

35. Holland, E.C.: Glioblastoma multiforme: the terminator. Proc. Natl. Acad. Sci. USA 97, 62426244 (2000)

36. Jain, R.K., di Tomaso, E., Duda, D.G., Loeffler, J.S., Sorensen, A.G., Batchelor, T.T.: Angiogenesis in brain tumours. Nat. Rev. Neurosci. 8, 610-622 (2007)

37. Jemal, A., Siegel, R., Ward, E., Murray, T., Xu, J., Thun, M.J.: Cancer statistics 2007. CA Cancer J. Clin. 57, 43-66 (2007)

38. Kansal, A.R., Torquato, S., Chiocca, E.A., Deisboeck, T.S.: Emergence of a subpopulation in a computational model of tumor growth. J. Theor. Biol. 207, 431-441 (2000a)

39. Kansal, A.R., Torquato, S., Harsh, G.I., Chiocca, E.A., Deisboeck, T.S.: Simulated brain tumor growth dynamics using a three-dimensional cellular automaton. J. Theor. Biol. 203, 367-382 (2000b)

40. Kansal, A.R., Torquato, S., Harsh, I.G., Chiocca, E.A., Deisboeck, T.S.: Cellular automaton of idealized brain tumor growth dynamics. Biosystems 55, 119-127 (2000c)

41. Kastan, M.B., Bartek, J.: Cell-cycle checkpoints and cancer. Nature 432, 316-323 (2004)

42. Kitano, H.: Computational systems biology. Nature 420, 206-210 (2002)

43. Lefranc, F., Brotchi, J., Kiss, R.: Possible future issues in the treatment of glioblastomas: special emphasis on cell migration and the resistance of migrating glioblastoma cells to apoptosis. J. Clin. Oncol. 23, 2411$2422(2005)$

44. Mansury, Y., Deisboeck, T.S.: The impact of "search precision" in an agent-based tumor model. J. Theor. Biol. 224, 325-337 (2003)

45. Mansury, Y., Kimura, M., Lobo, J., Deisboeck, T.S.: Emerging patterns in tumor systems: simulating the dynamics of multicellular clusters with an agent-based spatial agglomeration model. J. Theor. Biol. 219, 343-370 (2002)

46. Mellinghoff, I.K., Wang, M.Y., Vivanco, I., Haas-Kogan, D.A., Zhu, S., Dia, E.Q., Lu, K.V., Yoshimoto, K., Huang, J.H., Chute, D.J., Riggs, B.L., Horvath, S., Liau, L.M., Cavenee, W.K., Rao, P.N., Beroukhim, R., Peck, T.C., Lee, J.C., Sellers, W.R., Stokoe, D., Prados, M., Cloughesy, T.F., Sawyers, C.L., Mischel, P.S.: Molecular determinants of the response of glioblastomas to EGFR kinase inhibitors. N. Engl. J. Med. 353, 2012-2024 (2005)

47. Miners, J.O., Smith, P.A., Sorich, M.J., McKinnon, R.A., Mackenzie, P.I.: Predicting human drug glucuronidation parameters: application of in vitro and in silico modeling approaches. Annu. Rev. Pharmacol. Toxicol. 44, 1-25 (2004)

48. Mischel, P.S., Cloughesy, T.F.: Targeted molecular therapy of GBM. Brain Pathol. 13, 52-61 (2003)

49. Mohamed, A., Zacharaki, E.I., Shen, D., Davatzikos, C.: Deformable registration of brain tumor images via a statistical model of tumor-induced deformation. Med. Image Anal. 10, 752-763 (2006)

50. Morrison, P.F., Laske, D.W., Bobo, H., Oldfield, E.H., Dedrick, R.L.: High-flow microinfusion: tissue penetration and pharmacodynamics. Am. J. Physiol. 266, R292-R305 (1994)

51. Nathoo, N., Chahlavi, A., Barnett, G.H., Toms, S.A.: Pathobiology of brain metastases. J. Clin. Pathol. 58, 237-242 (2005)

52. Pallud, J., Mandonnet, E., Duffau, H., Kujas, M., Guillevin, R., Galanaud, D., Taillandier, L., Capelle, L.: Prognostic value of initial magnetic resonance imaging growth rates for World Health Organization grade II gliomas. Ann. Neurol. 60, 380-383 (2006)

53. Sanga, S., Sinek, J.P., Frieboes, H.B., Ferrari, M., Fruehauf, J.P., Cristini, V.: Mathematical modeling of cancer progression and response to chemotherapy. Expert Rev. Anticancer Ther. 6, 1361-1376 (2006)

54. Sanga, S., Frieboes, H.B., Zheng, X., Gatenby, R., Bearer, E.L., Cristini, V.: Predictive oncology: a review of multidisciplinary, multiscale in silico modeling linking phenotype, morphology and growth. Neuroimage 37(Suppl 1), S120-S134 (2007)

55. Schmitz, J., Kansal, A.R., Torquato, S.: A cellular automaton model of brain tumor treatment and resistance. J. Theor. Med. 4, 223-239 (2002)

56. Sinek, J., Frieboes, H., Zheng, X., Cristini, V.: Two-dimensional chemotherapy simulations demonstrate fundamental transport and tumor response limitations involving nanoparticles. Biomed. Microdevices 6, 297-309 (2004) 
57. Stamatakos, G.S., Antipas, V.P., Uzunoglu, N.K.: A spatiotemporal, patient individualized simulation model of solid tumor response to chemotherapy in vivo: the paradigm of glioblastoma multiforme treated by temozolomide. IEEE Trans. Biomed. Eng. 53, 1467-1477 (2006a)

58. Stamatakos, G.S., Antipas, V.P., Uzunoglu, N.K., Dale, R.G.: A four-dimensional computer simulation model of the in vivo response to radiotherapy of glioblastoma multiforme: studies on the effect of clonogenic cell density. Br. J. Radiol. 79, 389-400 (2006b)

59. Stamatakos, G.S., Zacharaki, E.I., Makropoulou, M.I., Mouravliansky, N.A., Marsh, A., Nikita, K.S., Uzunoglu, N.K.: Modeling tumor growth and irradiation response in vitro-a combination of high-performance computing and web-based technologies including VRML visualization. IEEE Trans. Inf. Technol. Biomed. 5, 279-289 (2001)

60. Stupp, R., Hegi, M.E., van den Bent, M.J., Mason, W.P., Weller, M., Mirimanoff, R.O., Cairncross, J.G.: Changing paradigms-an update on the multidisciplinary management of malignant glioma. Oncologist 11, 165-180 (2006)

61. Swanson, K.R., Alvord, E.C. Jr., Murray, J.D.: A quantitative model for differential motility of gliomas in grey and white matter. Cell Prolif. 33, 317-329 (2000)

62. Swanson, K.R., Alvord, E.C. Jr., Murray, J.D.: Virtual brain tumours (gliomas) enhance the reality of medical imaging and highlight inadequacies of current therapy. Br. J. Cancer 86, 14-18 (2002a)

63. Swanson, K.R., Alvord, E.C. Jr., Murray, J.D.: Quantifying efficacy of chemotherapy of brain tumors with homogeneous and heterogeneous drug delivery. Acta Biotheor. 50, 223-237 (2002)

64. Swanson, K.R., Bridge, C., Murray, J.D., Alvord, E.C. Jr.: Virtual and real brain tumors: using mathematical modeling to quantify glioma growth and invasion. J. Neurol. Sci. 216, 1-10 (2003)

65. Tracqui, P., Cruywagen, G.C., Woodward, D.E., Bartoo, G.T., Murray, J.D., Alvord, E.C. Jr.: A mathematical model of glioma growth: the effect of chemotherapy on spatio-temporal growth. Cell Prolif. 28, 17-31 (1995)

66. Walker, D.C., Hill, G., Wood, S.M., Smallwood, R.H., Southgate, J.: Agent-based computational modeling of wounded epithelial cell monolayers. IEEE Trans. Nanobioscience 3, 153-163 (2004)

67. Wang, Z., Zhang, L., Sagotsky, J., Deisboeck, T.S.: Simulating non-small cell lung cancer with a multiscale agent-based model. Theor. Biol. Med. Model 4, 50 (2007)

68. Wein, L.M., Wu, J.T., Ianculescu, A.G., Puri, R.K.: A mathematical model of the impact of infused targeted cytotoxic agents on brain tumours: implications for detection, design and delivery. Cell Prolif. 35, 343-361 (2002)

69. Wessels, J.T., Busse, A.C., Mahrt, J., Dullin, C., Grabbe, E., Mueller, G.A.: In vivo imaging in experimental preclinical tumor research-a review. Cytometry A 71, 542-549 (2007)

70. Wishart, D.S., Yang, R., Arndt, D., Tang, P., Cruz, J.: Dynamic cellular automata: an alternative approach to cellular simulation. In Silico Biol. 5, 139-161 (2005)

71. Wolfram, S.: A New Kind of Science. Wolfram Media, Champaign, IL (2002)

72. Zhang, L., Athale, C.A., Deisboeck, T.S.: Development of a three-dimensional multiscale agent-based tumor model: simulating gene-protein interaction profiles, cell phenotypes and multicellular patterns in brain cancer. J. Theor. Biol. 244, 96-107 (2007)

73. Zheng, X., Wise, S.M., Cristini, V.: Nonlinear simulation of tumor necrosis, neo-vascularization and tissue invasion via an adaptive finite-element/level-set method. Bull. Math. Biol. 67, 211-259 (2005) 